\title{
An interface study of crystalline Fe/Ge multilayers grown by molecular beam epitaxy
}

\author{
S. Tari* \\ Department of Physics, Izmir Institute of Technology, Izmir, Turkey
}

\section{A R T I C L E I N F O}

\section{Article history:}

Received 22 November 2010

Received in revised form 6 December 2010

Accepted 7 December 2010

Available online 17 December 2010

\section{Keywords:}

Spintronics

Interface

Epitaxy

Intermixing

Crystalline

MBE

XPS

\begin{abstract}
A B S T R A C T
$\mathrm{Fe} / \mathrm{Ge}$ multilayers were grown on single crystal $\mathrm{Ge}(001)$ substrates by molecular beam epitaxy. The structural, electronic and magnetic properties of $\mathrm{Fe} / \mathrm{Ge}$ have been studied. The analysis shows that $\mathrm{Fe}$ grows in a layer-by-layer epitaxial growth mode on $\mathrm{Ge}(001)$ substrates at $150^{\circ} \mathrm{C}$ and no intermixing has been observed. Growth of a crystalline Ge film at $150^{\circ} \mathrm{C}$ on a single crystal Fe film has been observed. At this temperature Ge films grow by means of the island growth mode according to reflection of high energy electron diffraction patterns. Fe layers of $36 \mathrm{~nm}$ thickness, deposited at $150^{\circ} \mathrm{C}$ on $\mathrm{Ge}(001)$ substrates, show two magnetization reversal values indicating the growth of Fe in two different crystal orientations. $36 \mathrm{~nm}$ thick $\mathrm{Fe}$ and $\mathrm{Ge}$ layers grown at $150^{\circ} \mathrm{C}$ in $\mathrm{Ge} / \mathrm{Fe} / \mathrm{Ge} / \mathrm{Fe} / \mathrm{Ge}(001)$ sequence shows ferromagnetic behavior, however, the same structure grown at $200^{\circ} \mathrm{C}$ shows paramagnetic behavior.
\end{abstract}

(c) 2010 Elsevier B.V. All rights reserved.

\section{Introduction}

Interfaces between ferromagnetic (FM) metals and semiconductors (SC) attract much interest because of their potential applications in electronic and "spintronic" devices [1]. They show novel structural and magnetic properties [2-4]. The growth of transition metals on SC generally results in intermixing which alters the magnetic properties of the heterostructure. The small mismatch, $\sim 1.3 \%$, between the lattice constant of $\mathrm{Ge}$ and twice the unit cell constant of bcc Fe makes it possible to grow single crystalline Fe films on $\mathrm{Ge}(001)$. Fe has been grown on $\mathrm{Ge}(001)$ surfaces at room temperature as well as at the elevated temperature of $150^{\circ} \mathrm{C}[5,6]$. Ultra thin $\mathrm{Fe} / \mathrm{Ge}$ multilayers were grown recently by the sputtering technique [7-9] and structural and magnetic properties have been studied. Ge layers were found to be amorphous. It is a challenge to grow epitaxial high quality, single crystal Ge films on single crystal Fe layers at $150^{\circ} \mathrm{C}$, because the normal growth temperature of $\mathrm{Ge}$ is about $600^{\circ} \mathrm{C}$. At about $200^{\circ} \mathrm{C}$ Fe starts to diffuse into Ge and a thick intermixed layer occurs, altering the magnetic and electronic properties. Therefore, Ge has to be grown at much lower temperatures to prevent intermixing.

$\mathrm{Ge}$ is a promising semiconductor for spintronics applications. Recently there has been much research on the injection of spin

\footnotetext{
* Tel.: +90 232750 7711; fax: +90 2327507707.

E-mail addresses: stari1@uic.edu, suleymantari@iyte.edu.tr
}

polarized current from a ferromagnetic layer into Ge films [10-12]. This study has resulted in a better comprehension of the growth of ferromagnetic/semiconductor heterostructures, namely $\mathrm{Ge} / \mathrm{Fe}$. $\mathrm{Fe}$ and Ge layers as well as Fe/Ge multilayers were deposited on $\mathrm{Ge}(001)$ substrates by molecular beam epitaxy (MBE) at 150 and $200^{\circ} \mathrm{C}$ and the resulting structural, magnetic and electronic properties have been studied.

\section{Experiment}

All layers were grown on n-type $\mathrm{Ge}(001)$ substrates using a Riber MBE-2300 system. Details of the wafer cleaning process can be found in a previous article [13]. After transfer to the growth chamber, the wafers were heated up to $250{ }^{\circ} \mathrm{C}$ and held there for $30 \mathrm{~min}$, and then held at $500^{\circ} \mathrm{C}$ for another $30 \mathrm{~min}$. This procedure produced smooth $(2 \times 1)$ reconstructed Ge surfaces. The background pressure was lower than $10^{-9}$ Torr at all times. The growth rates of the Fe and Ge films were 0.4 and $0.26 \AA /$ s, respectively, as determined by transmission electron microscopy (TEM). The thicknesses indicated in this article were determined from the growth time assuming constant deposition rates.

The X-ray photoelectron spectroscopy (XPS) measurements were performed with a SSX-100 spectrometer with a monochromatic and focused $\mathrm{Al} \mathrm{K \alpha} \mathrm{X}$-ray source. Fe and Ge high resolution peak scans were recorded with a $600 \mu \mathrm{m}$ spot diameter and $50 \mathrm{eV}$ pass energy. The corresponding energy resolution was $0.9 \mathrm{eV}$ as measured by the full width at half-maximum (FWHM) of the Au4f 

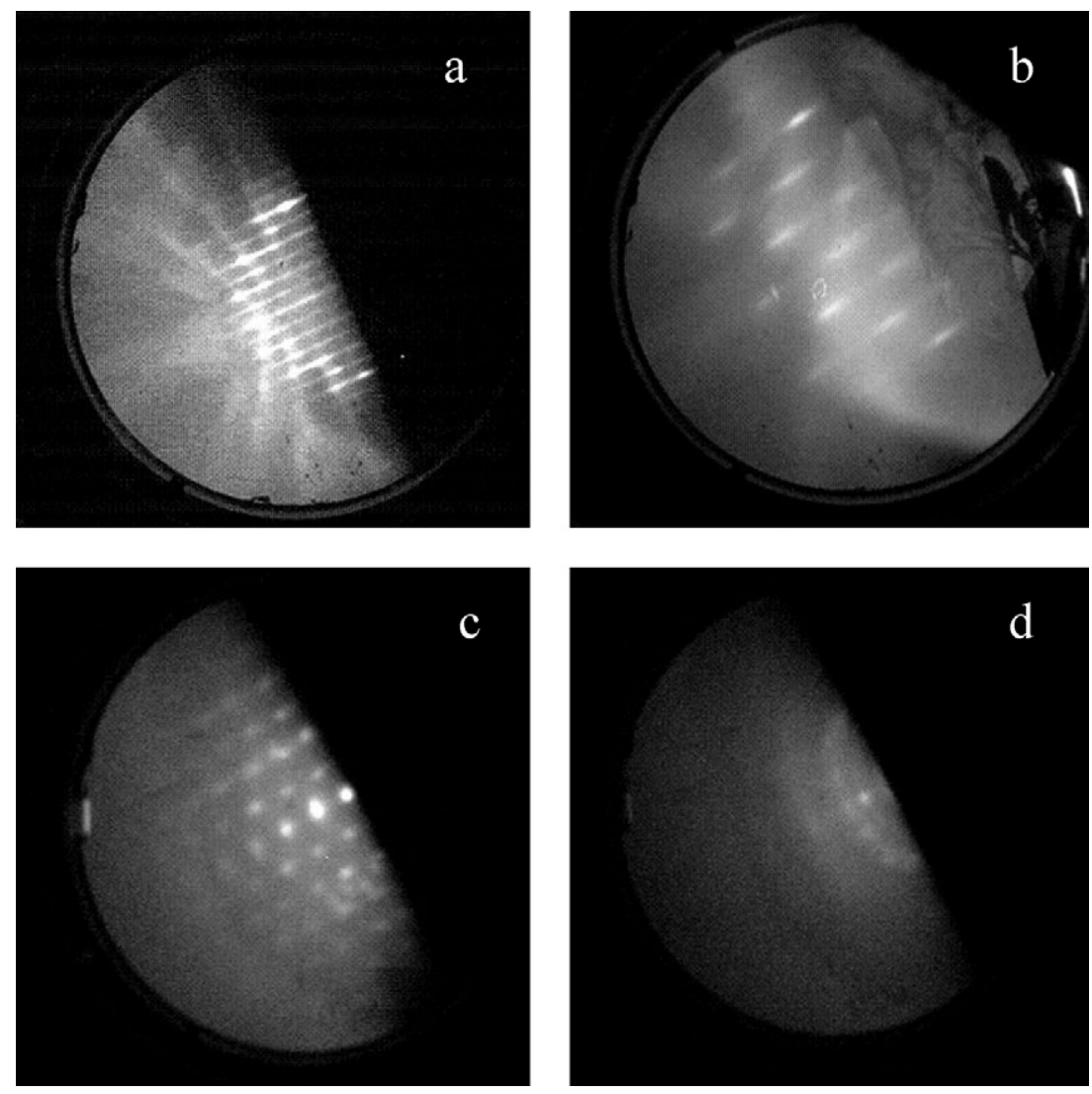

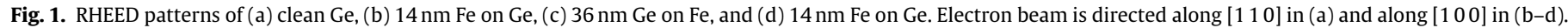
Both $\mathrm{Fe}$ and $\mathrm{Ge}$ layers are grown at $150^{\circ} \mathrm{C}$.

core level. The surface of the Ge wafer and the growth of Fe and Ge layers were monitored in situ by reflection of high energy electron diffraction (RHEED). The magnetic hysteresis loops were recorded by a superconducting quantum interference device (SQUID) at room temperature.

\section{Results}

The first, $\mathrm{Fe} / \mathrm{Ge}(001)$, and the second, $\mathrm{Ge} / \mathrm{Fe}$, interfaces of $\mathrm{Ge} / \mathrm{Fe} / \mathrm{Ge}(001)$ structure are represented by $\mathrm{Fe} / \mathrm{Ge}$ and $\mathrm{Ge} / \mathrm{Fe}$, respectively. The RHEED patterns are shown in Fig. 1. Fig. 1a shows the surface of the chemically cleaned and heated $\mathrm{Ge}(001)$ wafer before Fe growth. The long and bright streaks are characteristic of an atomically smooth $(2 \times 1)$ reconstructed surface. After $14 \mathrm{~nm}$ of Fe growth at $150^{\circ} \mathrm{C}$ the $(2 \times 1)$ surface reconstruction is transformed into the $(1 \times 1)$ surface of the bcc Fe(001) film as shown in Fig. 1b. The long and bright streaks in the pattern indicate that the Fe surface is quite smooth and the growth is via a layer by layer mode. Fig. 1c shows the pattern for a $36 \mathrm{~nm}$ Ge film deposited at $150^{\circ} \mathrm{C}$ on a $14 \mathrm{~nm}$ thick Fe layer. The spotty pattern indicates the presence of a rough surface, which implies that Ge grows on Fe via the island mode. This pattern at the same time indicates crystalline Ge growth on Fe film. Crystalline Ge growth is presented here for the first time to the best of our knowledge. The initial growth of $\mathrm{Ge}$ layer was not clearly seen in the RHEED patterns. The pattern due to Ge (Fig. 1c) disappeared when the second Fe layer was grown on the rough Ge surface as shown in Fig. 1d. The rings in the pattern are due to amorphous growth of Fe layer on Ge.

XPS spectra were recorded at various stages of growth at both interfaces. The result of the XPS analysis of the Fe/Ge interface has been published in our previous publication [13] and did not provide evidence of a significant reaction between Fe and Ge at $150^{\circ} \mathrm{C}$.
The change in the peak areas confirmed that the growth mode of $\mathrm{Fe}$ is layer by layer when grown at $150^{\circ} \mathrm{C}$. However, the sample grown at $200^{\circ} \mathrm{C}$ resulted in some reaction or interdiffusion at the interface which altered the magnetic properties by increasing the coercive field $\left(H_{c}\right)$ of the Fe layer. TEM diffraction patterns and cross sectional micrographs confirmed these results [13].

For the $\mathrm{Ge} / \mathrm{Fe}$ interface the peak areas of the $\mathrm{Ge} 2 \mathrm{p}$ and $\mathrm{Fe} 2 \mathrm{p}$ peaks as a function of Ge thickness are shown in Fig. 2. The solid lines were obtained by least squares fits of $I_{0} \exp (-d / \lambda)$ for the bottom Fe layer and $I_{\infty}(1-\exp (-d / \lambda))$ for the Ge overlayer where $\lambda$ is the attenuation length. The peak areas of Ge 2 p follow the exponential curve reasonably well suggesting a uniform coverage of the

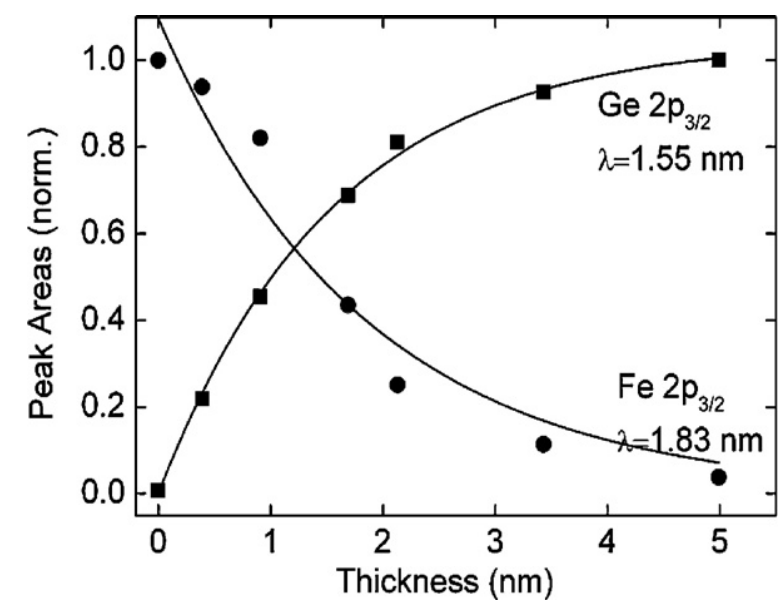

Fig. 2. Peak areas of Ge2p and Fe2p core level peaks as a function of Ge thickness on Fe layer, deposited at $150^{\circ} \mathrm{C}$. 


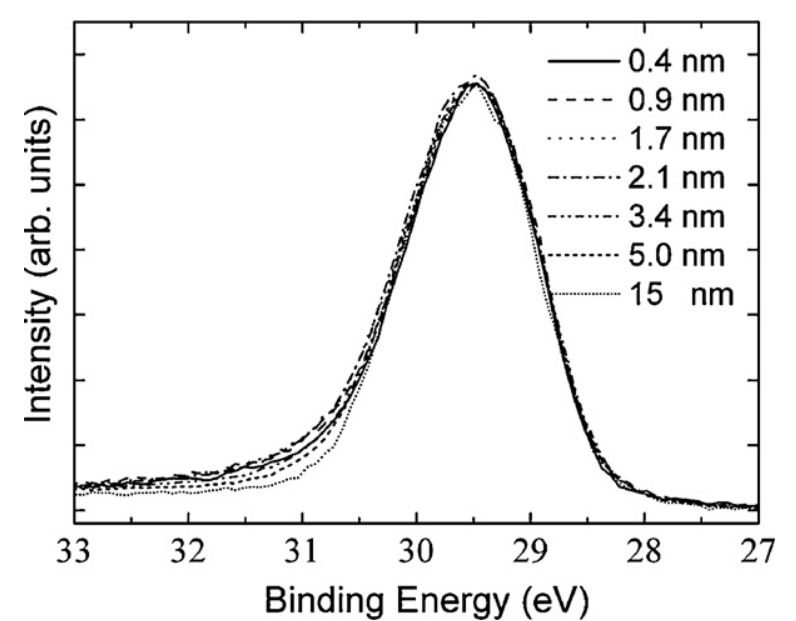

Fig. 3. Normalized intensities of Ge3d peak for various thickness of Ge on Fe layer.

Ge on Fe. However, the peak areas of Fe $2 p$ especially for thin Ge layers, do not follow the curves as well for thicknesses up to $2 \mathrm{~nm}$. The attenuation lengths were found to be 1.55 and $1.83 \mathrm{~nm}$ for Ge $2 \mathrm{p}$ and Fe $2 \mathrm{p}$, respectively. Although the Ge data follow the fitted curve quite well, $\lambda$ for both Ge $2 \mathrm{p}$ and Fe $2 \mathrm{p}$ values are larger than expected for the energy of these electrons compared with the universal curve of Seah and Dench [14]. The theoretical attenuation lengths of Ge 2p and Fe 2p are 0.42 and $0.72 \mathrm{~nm}$, respectively. These large experimental attenuation lengths may be associated with the reacted layers and intermixing at the interface of Ge/Fe (see XPS data below) as well as the possible non-uniform Ge surface. We have not been able to get better attenuation lengths including a parameter to take nonuniform interface of $\mathrm{Ge} / \mathrm{Fe}$ into account in the fitting process. Therefore, we propose, according to the XPS analysis the growth of thin Ge on Fe can be better explained by layer by layer growth. The Rheed pattern in Fig. 1c indicates that the growth is in islanding mode, however, that pattern represents

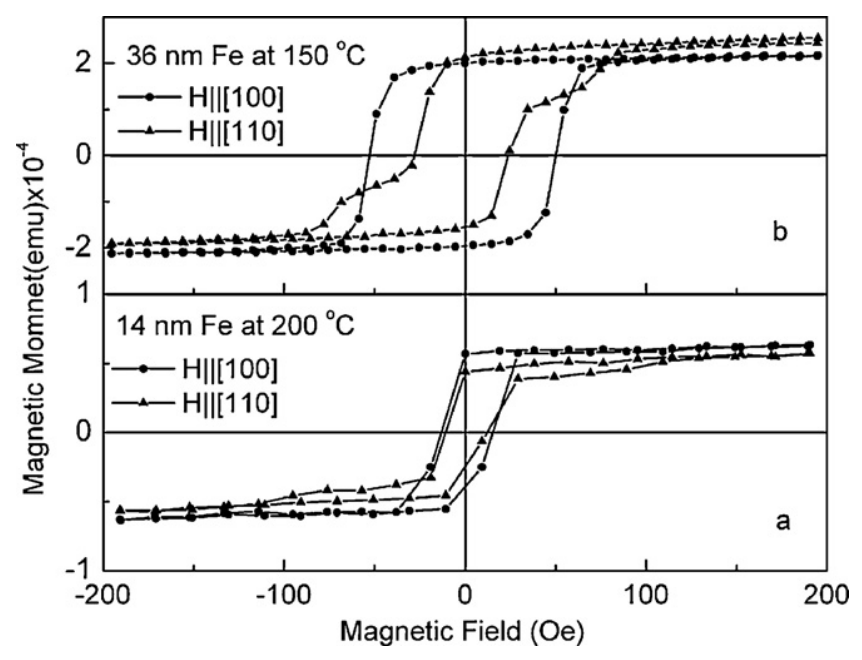

Fig. 5. M-H loops of Fe layer on $\mathrm{Ge}(001)$.(a) $14 \mathrm{~nm}$ deposited at $200^{\circ} \mathrm{C}$ and (b) $36 \mathrm{~nm}$ deposited at $150^{\circ} \mathrm{C}$. $\mathrm{H}$ is applied along both [ 100 ] and [ 110 ] directions.

a thickness of $40 \mathrm{~nm}$ of Ge growth on Fe. Moreover, it is common to find large errors for attenuation lengths experimentally [15].

In order to understand whether there is an intermixing, the peak intensities of $\mathrm{Ge} 3 \mathrm{~d}$ peaks are scaled to the same level for comparison. Normalized intensities of Ge 3d peaks are shown in Fig. 3. There is a slight decrease in the intensity of the peak at about $31 \mathrm{eV}$ as the thickness of $\mathrm{Ge}$ increases. In addition this peak becomes more symmetric which is the case for semiconductors. Although this change seems small, it might indicate the existence of a reaction or intermixing. Therefore, to investigate further we fitted the Ge 3d peaks for various Ge coverage as shown in Fig. 4. The spin-orbit splitting for $\mathrm{Ge}$ is $0.62 \mathrm{eV}$ and was held constant in the fitting process. The spin-orbit splitting is not resolved because the FWHM of the Ge 3d peak is about $1 \mathrm{eV}$. The Shirley background was subtracted from the spectra before the fit, and peaks were fitted with mixed doublets

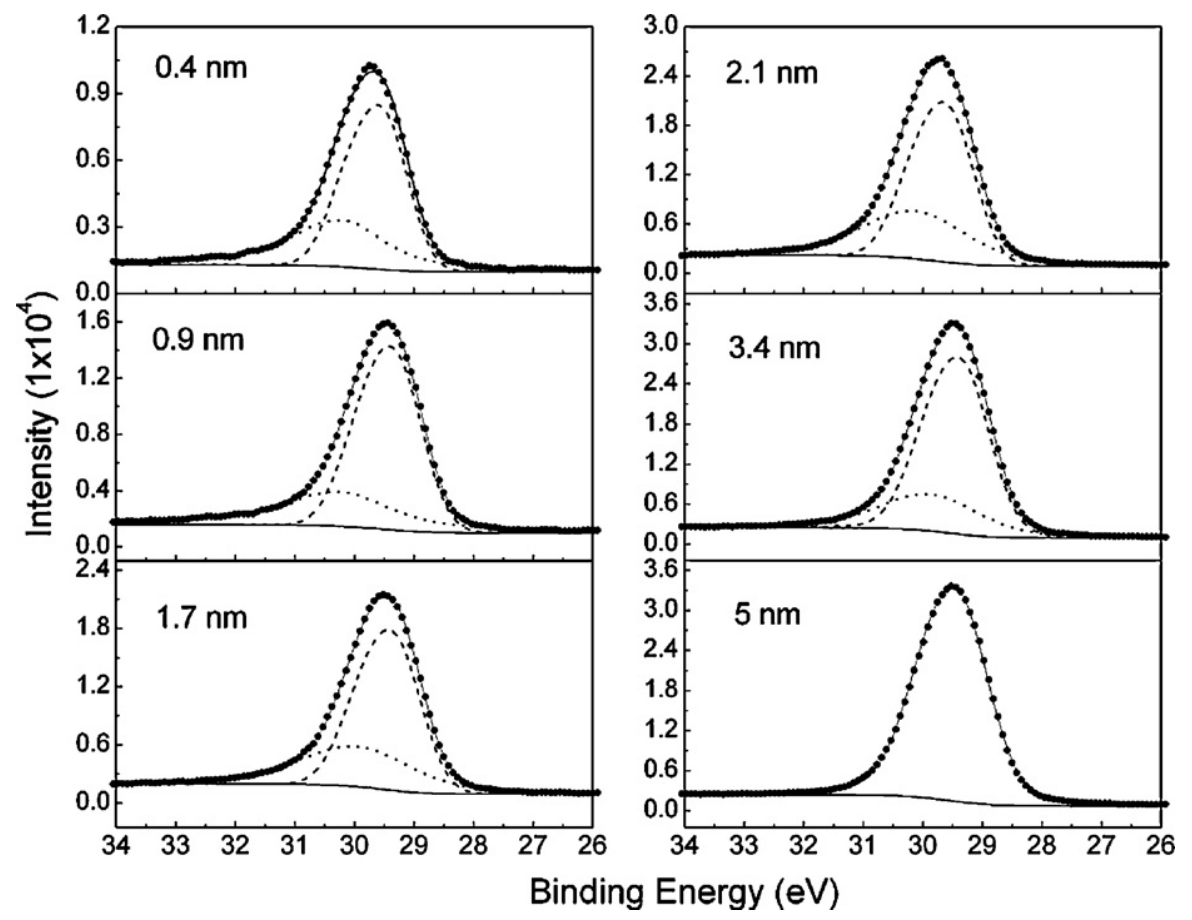

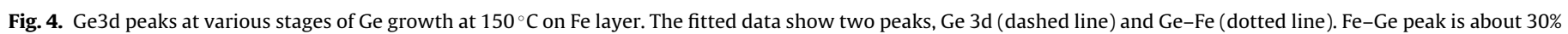
of the total peak intensity. 


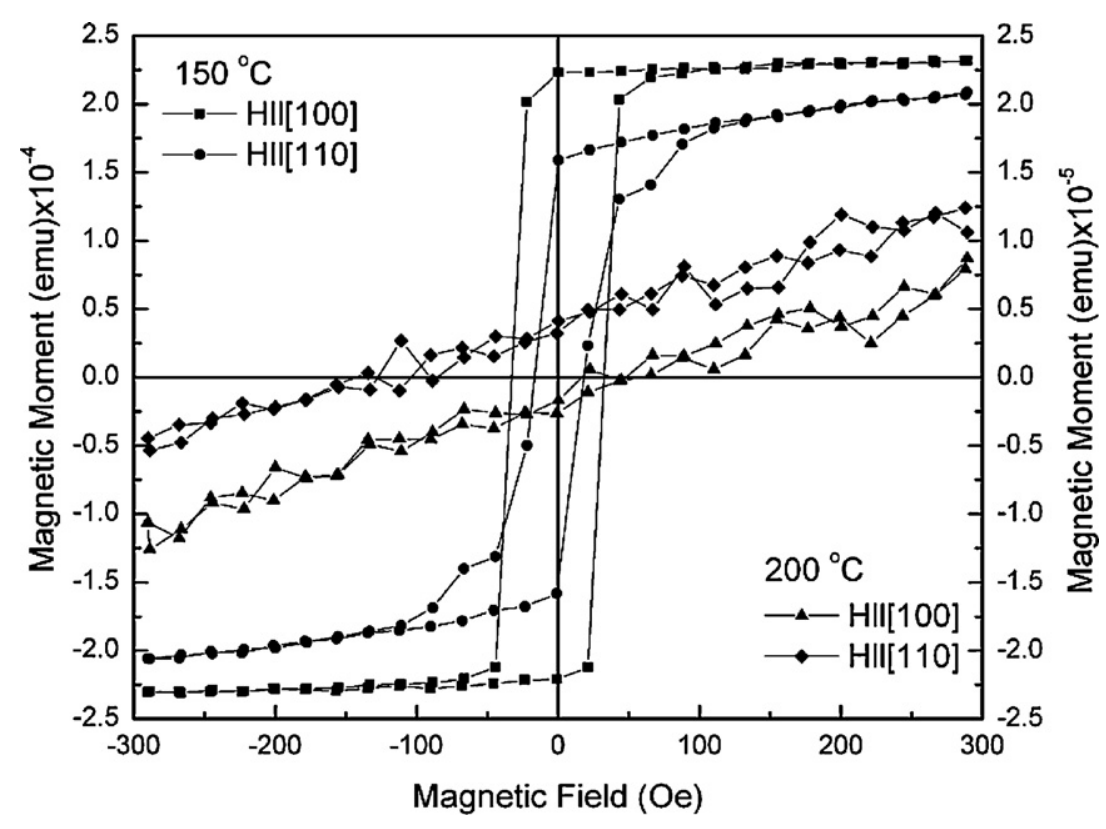

Fig. 6. $\mathrm{M}-\mathrm{H}$ loops of $\mathrm{Ge} / \mathrm{Fe} / \mathrm{Ge} / \mathrm{Fe} / \mathrm{Ge}(001)$ structures grown at 150 and $200{ }^{\circ} \mathrm{C}$. Both $\mathrm{Fe}$ and Ge thicknesses are $36 \mathrm{~nm}$ for each layer.

(Gaussian + Lorentzian) peaks. We had to use two mixed doublets. Quantitative analysis of the peak fits show that for all Ge layers the contribution of the second peak is about $30 \%$ which is large enough to predict the existence of an intermixing. At the same time a small fraction of this value $(\sim 10 \%)$ may be due to the Ge and Fe bonding at the interface. For a $5 \mathrm{~nm}$ thick Ge one mixed doublet peak was sufficient to fit the peak reasonably well. Although XPS results suggest some intermixing, they are not sufficient by themselves to determine the extent of the intermixing. A TEM study of the Fe/Ge interface might be helpful in clarifying the XPS data.

Magnetic hysteresis loops (M-H) were recorded with a Squid magnetometer at room temperature. The magnetic field $(H)$ was applied along both the easy [100] and the hard [110] axes. The applied magnetic field direction refers to the direction of easy and hard axes of the magnetic layer. In our case magnetic layer is Fe which has easy and hard axes in [ 100$]$ and [ 110$]$, respectively. It should be noted that both Fe and Ge have cubic crystal structure and growth is epitaxial. The $\mathrm{M}-\mathrm{H}$ loops for a $14 \mathrm{~nm}$ thick Fe film grown on $\mathrm{Ge}(001)$ substrate at $200^{\circ} \mathrm{C}$ is shown in Fig. 5a. A capping layer of $5 \mathrm{~nm}$ was grown on Fe in this case to prevent the oxidation of the Fe layer. As can be seen, the loop has a square shape which indicates that the Fe layer is crystal with large grain sizes resulting in large magnetic domains. The magnetization reversal process is due completely to domain walls motion. The $H_{C}$ is about 10 Oersted (Oe), which is a characteristic value of Fe layers grown by MBE. The growth technique affects the magnetic properties to a large extent. When $H$ is applied along the hard axis a small step was observed at about 90 Oe. Fig. 5b shows the M-H loops of a $36 \mathrm{~nm}$ thick Fe layer grown at $150^{\circ} \mathrm{C}$. Again this Fe layer shows similar behavior. $H_{c}$ is larger because the thickness of Fe is almost double that of Fe grown at $150^{\circ} \mathrm{C}$. It is well known that $H_{c}$ of Fe layer increases with increasing thickness [16]. It is also possible that the intermixing (see XPS data) might result in an increase of $H_{c}$. Intermixing may create defects and a rough interface which might pin the domain walls hence increase $H_{c}$. When a magnetic field was applied along [1 10 ], the Fe layer showed two clear switching magnetic reversal values resulting from the crystalline anisotropy. The reason for this step formation is associated with the growth mechanism for thicker Fe [16]. This indicates that Fe layers grow with two different orientations on Ge wafer as the Fe thickness increases. Large magnetic domains are aligned along the crystalline directions. The magnetization reversal also has a contribution from rotation of the magnetization along with domain wall motion.

We have also grown multilayers in the form of $\mathrm{Ge} / \mathrm{Fe} / \mathrm{Ge} / \mathrm{Fe} / \mathrm{Ge}(001)$ at both 150 and $200^{\circ} \mathrm{C}$ in order to investigate the effect of temperature on the magnetic properties. The thickness of the $\mathrm{Fe}$ and Ge layers are $36 \mathrm{~nm}$. The $\mathrm{M}-\mathrm{H}$ loops are shown in Fig. 6. The magnetic field was applied along both the [ 100$]$ and the [1 10 ] directions. The multilayers grown at $150^{\circ} \mathrm{C}$ show ferromagnetic behavior with a decrease in $H_{c}$ from 54 to 33 Oe as compared with the Fe layer. This decrease in $H_{c}$ may be associated with the structural changes in the second Fe layer which was found to be polycrystalline (see Fig. 1d) and the possible intermixing at the four interfaces of Fe/Ge multilayers. It is well known that structural changes affect the magnetic behavior of the ferromagnetic (FM) layers to a large extent. Although not as strong as in the Fe layer, the step was also seen in multilayers when $H$ was applied along the hard axis. However, we cannot associate this change in the step to any coupling mechanism between Fe layers at this point because the thickness of the Ge layer is $36 \mathrm{~nm}$, which is too large for coupling of two Fe layers. Multilayers structure grown at $200^{\circ} \mathrm{C}$ show paramagnetic behavior (which is FM for Fe layer). No change has been observed when $H$ was applied along the hard axis or any other angles. It can be inferred that at $200^{\circ} \mathrm{C}$ there is a total intermixing between $\mathrm{Fe}$ and $\mathrm{Ge}$ that diminishes the magnetization of the Fe layers. This result shows that growth of ferromagnetic sandwiches of $\mathrm{Fe} / \mathrm{Ge}$ heterostructures for spintronics applications should be carried at temperatures not higher than of about $150^{\circ} \mathrm{C}$.

\section{Conclusions}

Fe grows in layer by layer growth mode on a $\mathrm{Ge}(001)$ substrate at $150^{\circ} \mathrm{C}$ and no intermixing has been observed at the $\mathrm{Fe} / \mathrm{Ge}(001)$ interface. A crystalline Ge film was grown on $\mathrm{Fe} / \mathrm{Ge}(001)$ at $150^{\circ} \mathrm{C}$ for the first time according to the Rheed pattern and the growth occurs via an island mode. XPS peak area analysis indicates a small intermixing at the $\mathrm{Ge} / \mathrm{Fe}$ interface. Magnetic characterization of a $36 \mathrm{~nm}$ thick Fe film grown at $150^{\circ} \mathrm{C}$ on a $\mathrm{Ge}(001)$ substrate shows two switching magnetization reversal values along the hard axis. The $\mathrm{Ge} / \mathrm{Fe} / \mathrm{Ge} / \mathrm{Fe} / \mathrm{Ge}(001)$ structure grown at $150^{\circ} \mathrm{C}$ is totally ferromagnetic whereas the same structure grown at $200^{\circ} \mathrm{C}$ is 
paramagnetic, which indicates a intermixing of Fe and Ge heterostructures.

\section{Acknowledgement}

I would like to thank Prof. S. Sivananthan for giving me the opportunity to work in the Microphysics Laboratory at the University of Illinois at Chicago.

\section{References}

[1] G.A. Prinz, in: B. Heinrich, J.A.C. Bland (Eds.), Ultrathin Magnetic Structures II, Springer, New York, 1994, p. 35.

[2] R. Fiederling, M. Kelm, G. Reuscher, W. Ossan, G. Schmidt, A. Waag, L.W. Molenkamp, Nature 402 (1999) 787.

[3] Y.B. Xu, E.T.M. Kernohan, D.J. Freland, A. Ercole, M. Tselepi, J.A.C. Bland, Phys. Rev. B 58 (1998) 890.
[4] E.M. Kneedler, B.T. Jonker, P.M. Thibado, R.J. Wagner, B.V. Shanabrook, L.J. Whitman, Phys. Rev. B 56 (1997) 8163.

[5] P. Ma, P.R. Norton, Phys. Rev. B 56 (1997) 9881.

[6] G.W. Anderson, P. Ma, P.R. Norton, J. Appl. Phys. 79 (1996) 5641.

[7] S. Singh, S. Basu, M. Vedpathak, R.H. Kodama, R. Chitra, Y. Goud, Appl. Surf. Sci. 240 (2005) 251.

[8] Y.W. Liu, W.B. Mi, E.Y. Jiang, H.L. Bai, J. Appl. Phys. 102 (2007) 063712.

[9] S. Sigh, S. Basu, M. Gupta, Physica B 385-386 (2006) 653.

[10] Y. Zhou, M. Ogawa, M. Bao, W. Han, R.K. Kawakami, K.L. Wang, Appl. Phys. Lett. 94 (2009) 242104

[11] C. Shen, T. Trypiniotis, K.Y. Lee, S.N. Holmes, R. Mansell, M. Husain, V. Shah, X.V. Li, H. Kurebayashi, I. Farrer, C.H. de Groot, D.R. Leadley, G. Bell, E.H.C. Parker, T. Whall, D.A. Ritchie, C.H.W. Barnes, Appl. Phys. Lett. 97 (2010) 162104.

[12] En-Shao Liu, J. Nah, K.M. Varahramyan, E. Tutuc, Nanoletters 10 (2010) 3297.

[13] S. Tari, R. Sporken, T. Aoki, David J. Smith, V. Metlusko, K. AbuEl-Rub, S. Sivananthan, J. Vac. Sci. Technol. B 20 (4) (2002) 1586.

[14] M.P. Seah, W.A. Dench, Surf. Interface Anal. 1 (1979) 2.

[15] M. Cantoni, M. Riva, G. Isella, R. Bertacco, F. Ciccacci, J. Appl. Phys. 97 (2005) 093906.

[16] M. Cantoni, M. Riva, R. Bertacco, F. Ciccacci, Phys. Rev. B 74 (2006) 134415. 